\title{
Emotions and Clinical Ethics Support. A Moral Inquiry into Emotions in Moral Case Deliberation
}

\author{
Bert Molewijk • Dick Kleinlugtenbelt • \\ Scott M. Pugh • Guy Widdershoven
}

Published online: 15 September 2011

(C) Springer Science+Business Media B.V. 2011

\begin{abstract}
Emotions play an important part in moral life. Within clinical ethics support (CES), one should take into account the crucial role of emotions in moral cases in clinical practice. In this paper, we present an Aristotelian approach to emotions. We argue that CES can help participants deal with emotions by fostering a joint process of investigation of the role of emotions in a case. This investigation goes beyond empathy with and moral judgment of the emotions of the case presenter. In a moral case deliberation, the participants are invited to place themselves in the position of the case presenter and to investigate their own emotions in the situation. It is about critically assessing the facts in the case that cause the emotion and the related (moral) thoughts that accompany the emotion. It is also about finding the right emotion in a given situation and finding the right balance in dealing with that emotion. These steps in the moral inquiry give rise to group learning. It is a process of becoming open towards the perspectives of others, leading to new insights into what is an appropriate emotion in the specific situation. We show how this approach works in moral case deliberation. A physician presents a situation in which he is faced with a pregnant woman who is about to deliver multiple extremely premature
\end{abstract}

The views expressed in this case are those of Scott Pugh and his co-authors and do not necessarily reflect the official policy or position of the Department of the Navy, the Department of Defense, or the United States government.

B. Molewijk $(\bowtie) \cdot$ G. Widdershoven

Department of Medical Humanities (Division VI), EMGO Institute for Health and Care Research, VU University Medical Centre (VUmc), Van der Boechorst Straat 7, 1081 BT Amsterdam,

The Netherlands

e-mail: b.molewijk@vumc.nl

B. Molewijk · D. Kleinlugtenbelt

GGNet, Institute of Mental Health Care, Moral Deliberation Group (GGNet Expertise Centre),

Zutphen, The Netherlands

S. M. Pugh

Department of Pediatrics, US Naval Hospital, Naples, Italy 
infants at the threshold of viability. The moral deliberation of the case and the emotions therein leads to the participants' conclusion that "compassion" is a more adequate emotion than "sadness". The emotion "sadness" is pointed towards the tragedy that is happening to the woman. The emotion "compassion" is pointed towards the woman; it combines consideration and professional responsibility. Through the shift towards compassion, participants experienced more creativity and freedom to deal with the sad situation and to support the woman. The paper ends with an analysis and reflection on the deliberation process. In the conclusion we argue for more attention to emotions in clinical ethics support and offer some directions for doing this in the right way.

Keywords Emotions $\cdot$ Moral decision-making $\cdot$ Moral case deliberation $\cdot$ Clinical ethics support services $\cdot$ Sadness $\cdot$ Compassion

\section{Introduction}

Emotions play a significant role in the practice of clinical ethics support (CES). ${ }^{1}$ There are many different and sometimes opposite views on the relevance of emotions for CES (Molewijk et al. 2011). Sometimes emotions are seen as irrelevant for CES or even as factors that disturb or undermine CES. Moreover, emotions are often regarded as essentially different from the moral domain. In bioethics, we see a primary focus on rationality and rational reasoning (Walker 1998; Nussbaum 2001). Many ethical theories explicitly or implicitly are based upon a rational anthropology: human beings in general are understood as rational and reasonable beings (Schneider 1998; Walker 1998; Haidt 2001), and in case they are not, ethicists should try to make human beings more rational and reasonable. This rational anthropology accounts for mistrust of emotions in general (Damasio 2003), and an ambivalent attitude towards emotions within CES. Within the literature, little has been published on how one could or should deal with emotions in CES in a practical or methodological way.

Whatever one thinks about the relationship of emotions and CES: we are faced with their interrelatedness in every day practice and in every case which comes up for moral inquiry. Emotions play a role in the actual case, in the moral judgments and decision-making within that case, and in the process of discussing a case. Our view on emotions is inspired by Aristotle (Aristotle 1923; Nussbaum 2001; Solbakk 2006). Aristotle considers emotion and moral reasoning to be internally related. In Aristotle's Nichomachean Ethics (NE), emotions are referred to as important, because they move our thinking and acting (Molewijk et al. 2011). Through our emotions, we know what is important for us. Thus, emotions contain knowledge. This knowledge is not theoretical, but practical. It is developed through practice and tells us how to act. Moral wisdom is being able to have the right emotions and act on them in a concrete situation. Wisdom is not gained by discarding emotions, but through developing the right emotion, appropriately suited to the situation.

\footnotetext{
${ }^{1}$ CES is understood as any clinical ethics activity that is aiming at supporting dealing with moral issues and questions in different ways (e.g., identification, reflection, education, decision-making, policy). These activities can be performed out of different structures (e.g., ethics committees, ethics consultants, moral deliberation, reflection groups).
} 
Based on an Aristotelian view of emotions, this paper presents a clinical ethics case in which emotions played a significant role. It concerns a pediatrician on duty in the hospital being faced with a pregnant woman about to deliver multiple extremely premature infants at the threshold of viability. The case has been discussed during an international workshop on emotions and moral deliberation. The case was presented by the pediatrician. We will describe and reflect upon the process of moral case deliberation and the specific role that emotions played in the moral inquiry. Our aim with this paper is to exchange (reflections about) practical experiences concerning the dealing with emotions within CES, and to develop practical suggestions for dealing with emotions in a suitable way.

\section{Moral Case Deliberation}

Moral case deliberation (MCD) is a specific form of $\mathrm{CES}^{2}$ (Molewijk et al. 2008; Abma et al. 2009; Widdershoven \& Molewijk 2010). A MCD consists of a meeting with health care professionals, clients, managers and so forth, who systematically reflect on one of their moral questions within a concrete clinical case from their practice. Questions often relate to the care situation ("What should we consider as the morally right thing to do in this specific situation and how should we do it rightly?"). Yet, more philosophical or conceptual questions in concrete situations can also be investigated in a moral case deliberation (e.g., "What is respect?", or "What does understanding mean?"). According to our approach to moral case deliberation, the primary goal of a session is to create a critical and respectful moral inquiry into both the moral issues in a clinical ethics case and the participants' values and norms. Moral case deliberation aims at one or more of the following goals: (1) to reflect on the case and to improve the quality of care within that case; (2) to reflect on what it means to be a good professional and to enhance professionals' moral competencies, (3) to reflect on institutional or organizational issues and to improve the quality of care at that level (Abma et al. 2009).

An MCD usually takes 45 minutes to 2 hours and is facilitated by a trained facilitator. The facilitator, an ethicist or someone who is trained in clinical ethics and the use of specific conversation methods (e.g., the Dilemma Method or the Socratic Dialogue), does not give substantial advice and does not morally justify or legitimize a specific decision. The expertise of the facilitator consists in fostering an open and constructive dialogue among the participants, keeping an eye on the moral dimension of the case, supporting the joint reasoning process, and helping the group in planning actions in order to improve the quality of care (policy). Conversation methods are a key ingredient of moral case deliberation. These methods delineate several steps or phases in which the central moral question is investigated; they structure the reasoning and deliberation process (Steinkamp \& Gordijn 2003; Ashcroft et al. 2005; Kessels et al. 2009; Molewijk \& Ahlzen 2011). In this paper,

\footnotetext{
${ }^{2}$ MCD differs in some essential aspects with clinical ethics consultation (e.g., the ethics facilitation approach). For a brief description of some differences see Molewijk et al. (2011).
} 
the conversation method is based upon an Aristotelian approach to emotions. This method is described in more detail elsewhere (Molewijk et al. 2011).

\section{Case Presentation and Process of Deliberation}

The case we will discuss was presented during a 1.5 hour international workshop on "the role of emotions in moral deliberation" in which around 20 international participants from different disciplinary backgrounds participated. ${ }^{3}$ We will present a chronological summary of the case discussion. In Three Ways of Dealing with Emotions, we will reflect on some key elements related to the role of emotions in the process of moral deliberation.

\section{Three Ways of Dealing with Emotions}

During the start of the workshop, participants were asked individually to recall a situation which had bothered them and in which they had experienced a specific emotion. The participants were asked to write down the extremes and the more appropriate middle in dealing with this emotion. The following instruction was given:

"Remember a concrete situation that goes to your heart and in which you were engaged. Check which emotion you had in that situation. Consider for instance the following emotions: anger, apathy, love, sadness, loneliness, compassion, fear, disgust, boredom, pride or joy. Imagine you are in that situation again. Write down your answers to the following questions:

- What would you do when the emotion entirely leads you; in other words, when the emotion controls your behavior?

- What would you do when you totally repress the emotion; in other words, when the emotion is put aside?

- What would you do when you take the right attitude to the emotion; in other words, when you have found the middle way between both extremes in your action?"

Everyone did the exercise in a silent internal conversation and wrote down the three ways of dealing with emotions. After this, the facilitator asked who wanted to present his case. One of the participants said he would like to do that. He had recalled a situation in which he had been very sad. The situation still bothered him. It was a case in which a pregnant woman was about to deliver multiple extremely premature infants. The group decided to take this example for moral deliberation.

\footnotetext{
3 The case presenter gave his informed consent for publication of this case. With respect to the clinical case (i.e., the pregnant woman) we deleted any referrals to time and location. We also changed some parts of the case in order to prevent recognition.
} 
Connecting the Emotions to the Facts

MCD Facilitator (F) "What was the primary emotion?"

Case Presenter (CP) "Sadness. There were more emotions (such as fear and anger), but the most prominent and crucial emotion was sadness."

F "What was the concrete situation in which the sadness emerged and in what situation was it the strongest?"

CP "I was most sad for the mother, for the loss of the joyful and hopeful expectations about being pregnant and having children, and that she would unfairly have to deal with such a difficult situation and be asked to make decisions related to the dying process her children would experience (...). I felt profound sadness, as if I was the mother myself and that I medically could do nothing. Here the emotion was the strongest and connected with anger and fear. This also happened because of my own experience then: I recently had become a father. Furthermore, I was a young professional in training."

F "Can you read out the three ways of dealing with emotions that you just wrote down?"

CP 1 "Too much. Emotionally too involved in the situation of mother and the loss of the premature infants. My feeling of sadness would interfere with concentration and decision-making. Also, the emotion leads me too much when the mother is overwhelmed with grief and begins crying or asking questions with clear desperation and hope for a positive answer when there is none. In this situation, feeling her pain, I could make errors by framing my responses in a way that gives her at least partially what she wants to hear rather than being 100\% clear and honest. As a consequence, it could also make me appear unprofessional or incapable of handling the medical responsibility through the power of the emotion in the opinion of the mother or my colleagues.

2 Too little. Not acknowledging the extreme sadness of the situation and the effect it has not only on me, but certainly on the mother and other staff members. There is no recognition of the emotions of mother and of myself: no involvement. This would negatively impact my performance by making me appear cold and uncaring to the mother. It also could manifest itself in other unintended ways in my own life (poor sleep or appetite, arguments with spouse, and so forth).

3 The right attitude. A right balance between a caring attitude (being a human myself) and professional abilities (responsibility). It should be not too emotionally involved and not a too technical attitude. Then it is possible to perform my medical duties to the best of my abilities and yet provide a warm, caring attitude towards the mother."

F "Does the note with the more appropriate middle correspond with what you did in that situation?' 
CP "No: there was a little too much sadness, too much involvement with the patient. Therefore, I had too little sight on what I had to do as a professional. My discussions with the mother may not have been as clear and concise as they could have been because I was internally distracted by my own sadness and anger about the situation. I may have allowed some false hope as I wanted so badly to give the mother answers that could alleviate her pain."

During the presentation of the case by the case presenter (with the help of the facilitator) the focus was primarily on the facts of the case, the case presenter, what the case presenter (retrospectively) considered as too much, too little and the right attitude towards the emotion and the correspondence between the middle position and the facts of the situation. The facilitator also asked the other participants: "What do you want to know about this concrete situation with the pregnant woman so that you become able to put yourself in the situation of the case-owner and his emotion?"

Various questions were asked, concerning the factual situation of the pregnant woman (e.g., the treatment, the diagnosis, the prognosis) and of the case presenter (e.g., his thoughts and behavior in that specific situation). The case presenter informed the group about these facts and also noted that he worked in a neonatal intensive care unit as a pediatric resident in training and that he was responsible for the medical care. This information resulted in the following description of the case:

"I responded to an emergency on the Labor \& Delivery Ward. A woman was imminently going to deliver multiple extremely premature infants at the threshold of viability. Very quickly we had to discuss her wishes for how aggressive our resuscitation efforts should be, given that the prognosis was very poor. The father of the children was not available at the time, and the mother requested full resuscitative efforts. Some hours after the delivery, it became apparent that the infants were not responding to our exhaustive efforts to sustain their health, and the likelihood of death despite all of our interventions became imminent. For the first time in my training, I was given the responsibility of delivering the bad news to the mother and discussing the options for withholding and withdrawing further life-sustaining medical treatment for her children. At the same time, I was responsible for performing the technical duties associated with their medical care (as well as that of other premature infants in the NICU at that time).

It is rather difficult to characterize the myriad emotions I felt during this event. They included shock, fear, anger, guilt, exhaustion and above all others, profound sadness. Having recently become a father myself, I could not imagine how much more heightened and painful those same emotions were being experienced by the mother of the infants. The particular emotion of sadness was difficult to deal with for fear that it would negatively affect my abilities to perform my medical duties, and yet on a human level, seemed important to experience along with the mother." 
Participants Imagine Being in the Same Situation as the Case Presenter

Then the facilitator asked the participants $(\mathrm{P})$ : "Put yourself in the place of the example giver. Would you have the same emotion in this situation or another one?" Participants brought up different emotions: sadness, compassion, desperation, fear, and anxiety.

Several participants said they would feel sadness in this situation. They saw the situation as really sad for the mother. They could imagine that as a doctor they would also feel sad, because they would not be able to do anything for the mother and the children. One of the participants mentioned he had a different emotion. He said: "I feel compassion in this situation."

F "Can you tell us the facts that emerged compassion in you? What kinds of thoughts are connected with compassion?"

P "I put myself in the situation of the mother: something terrible has happened to her and to the infants during her pregnancy-her children will die and medically I can do nothing for her and her infants; she is not to blame and she can do nothing about it; then I feel compassion."

Imagining themselves in the situation, some participants mentioned emotions like fear.

F "Can you tell us the facts and thoughts that emerged fear in you?"

P "I would feel fear, because I would have to tell the mother that her infants will die and that I would not be able to do anything as a pediatrician. I would think: 'How should I tell this bad news to a desperate mother?' That would give me fear."

While talking about and further investigating these emotions, the case presenter and the other participants recognized compassion as the most suitable to the situation. Compassion implies being related to the mother, instead of identifying with the disaster which is happening to her. In the emotion of sadness, there seems to be a focus on the tragedy. In the emotion of compassion, there is more a focus on the mother. As a professional you are not able to cure or prevent the tragedy; you only can inform the mother about the facts of this tragedy. However, as a professional you are able to support the mother in dealing with that tragedy. Compassion opens more possibilities for openness towards both coping with the difficult situation and the professional relationship with the mother. Fear blocks action.

During the moral inquiry process on "compassion" some participants asked: "Is compassion an emotion or an excellence or a virtue?" During the answering of that question, participants started to refer to theories, concepts and opinions. Hence, the conversation slowly changed from a dialogue and moral inquiry into a discussion in which different statements and abstract knowledge claims were used alternately. The relationship with the concrete situation of the case disappeared. Such a discussion can be useful but is not appropriate within a moral case deliberation. Therefore, the facilitator redirected the process by asking the participants to return to the emotions in the case and focus on the accompanying thoughts. He specifically addressed the case presenter to reflect on the right middle ground for dealing with the situation. 
F "Was there in the middle a free attitude towards the emotion of sadness?"

$\mathrm{CP}$ "Not in the situation itself for me. It was an effort to stay in balance. However, the moral inquiry right now gives me more freedom. I think and experience that 'compassion' fits more with a free professional attitude. A free attitude towards the emotion allows me to acknowledge and empathize, but maintain composure, communicate clearly and honestly and provide proper care."

Another participant "Was there perhaps too much closeness in your sadness?

said

Did your involvement or concern with the mother cause a lack of appropriate professional distance?"

Another participant "What did you do with your sadness towards the mother?" asked the $\mathrm{CP}$

CP "I put my hand on her shoulder, so I showed my support and consolation."

Thus, the participants investigated in a concrete way what an appropriate emotion would be in the situation. Participants were looking for an emotion which combines a professional attitude with showing consideration and providing support and at the same time being honest about the medical facts.

\section{Lessons Learned}

In the last phase of the moral case deliberation, the participants reflected upon what they learned from the moral inquiry into the emotions. Participants referred to what they learned from the process of moral inquiry and what they learned about the emotion of sadness.

F "What were the lessons learned?"

The participants mentioned the following lessons:

- "Be reflective about these emotional situations."

- "It is possible to think in another way. It is possible to bring the emotions in agreement with the facts and with one's own life experiences."

- "Recognize and acknowledge the emotions of the different persons, such as the patient but also your own emotions."

- "Give words to the emotions."

- "Sometimes you have to just let there be sadness and experience it fully."

- "If you think about the goal of helping the mother and the children, then you can experience your emotion without letting it getting out of hand."

\section{Analysis of and Reflections on the Role of Emotions in Moral Deliberation}

The case description shows that within clinical ethics support one can critically reflect on emotions. By focusing on the emotion in the case, one can learn how to deal with emotions in practice and integrate them in moral life. In order to focus on the emotion and engage in a moral inquiry, one has to learn what one (as a facilitator and as a group) should not do. In the example, the facilitator did not demonstrate a counseling or caring attitude with respect to the case presenter. Too much empathy 
with the person's emotions may decrease critical thinking. Moral deliberation is not a counseling or peer-group session in which one person is "helped" and as a consequence functions as "the" object of moral inquiry. A moral deliberation involves a process of moral inquiry in which all participants assess their thinking, feeling and behaving as if they were in the same situation of the case presenter. So the case presenter is neither helped, nor morally judged about what he did or felt. All participants reflect on their own thinking, feeling and behaving. To put it succinctly: moral deliberation is primarily not about empathy but about moral inquiry. ${ }^{4}$

The main task of the facilitator was to facilitate the group into reflections on the emotion, dealing with the emotion and associated thoughts regarding the emotion. In the reflection process, the facts of the case were used as an important anchor for the inquiry process. In this way, emotions are not just individual and only subjective experiences which cannot be dealt with in a reasonable way. One can respect (the person who experiences) emotions and at the same time criticize them. ${ }^{5}$ Emotions are not taken for granted; they are seen as part of a complex chain of reasoning, interpretations and conclusions which can be assessed. During moral case deliberation, emotions are scrutinized through a process of asking questions.

Thus, it is possible to investigate the appropriateness of the way in which people deal with an emotion. The middle position can be morally assessed. The reason why a certain way of dealing with an emotion is (not) appropriate can also be investigated. The middle position emerges through reflection on what we consider an appropriate "sadness" or "compassion" in relation to the facts in that case that participants consider as important. For example, too much sadness could tempt the pediatrician to misrepresent medical facts or to present the facts differently in order to answer the woman's desperate search for hope. Moreover, certain emotions can be more appropriate than others. Thus, the group came to the conclusion that compassion is a better emotion than sadness in the case under consideration, because it does not imply identification with the woman, and enables a caring attitude towards her.

In situations of moral uncertainty, unease, or stress, emotions often are experienced as burdensome. Through the process of moral deliberation and reflection upon emotions and associated thoughts, one learns to relate to the emotions and the immediate appeal they make on us. This results in a situation in which emotions do not linea recta guide us; our free space and critical thinking increases.

\footnotetext{
${ }^{4}$ It is important to inform the participants, and especially the case presenter, beforehand what the aim of the moral case deliberation is (i.e., a moral inquiry into the moral question) and what they can expect to happen. Often this is not enough: one also has to inform the participants what they should not expect (e.g., counseling or helping an individual or looking for psychological clarifications of certain behavior and emotions).

${ }^{5}$ In order to scrutinize emotions and at the same time respect the MCD participants, two important features of moral case deliberation were acted upon: (1) people judge their emotions and their associated thoughts only after the process of imagining themselves as if they were in the situation of the case presenter; (2) criticism towards other participants' emotions and thoughts are processed through the process of asking questions (as opposed to making judgments).
} 
Finally, it is important to reflect upon what actually functions as "the case" within the moral case deliberation. Quite often, in clinical ethics case analysis or in medical ethics teaching situations, participants reflect upon the case in order to decide what is morally appropriate to do or to decide. During this reflection those who reflect (i.e., the participants of the moral case deliberation) position themselves outside of the case. In the example described above, the case was not a medical case of a pregnant woman and her emotions, and neither was it a case about a professional with his emotions, thoughts and decisions. During the deliberation process, the case became the vehicle for reflection on the emotions of all the participants. The focus was not on general ethical concepts, principles and professional codes, but on the experiences and the reasoning process of the participants in the group at that very moment.

To conclude, we asked the participants how they experienced this workshop on the role of emotions in moral case deliberation. The case presenter gave the following answer.

CP "In general terms, as a result of this exercise I have learned how important it is to know yourself and your values and to give time for inner reflection when faced with difficult situations. I also realize not only how emotions affect thoughts and actions, but conversely how my thoughts can affect my emotions. By keeping focus on the virtue I wish to achieve, my thoughts will help guide the proper inclusion of emotions into my actions. The freedom to experience the emotion while maintaining a grounded state comes directly from my thoughts about the emotion itself.

In this specific example, I believe finding the balance of including emotions into my thoughts and actions without allowing them to negatively impact my primary goal of doing good and helping others is paramount. If confronted with a similar situation in the future, I will continue to be open and honest with my own emotions, but plan to allow myself private time to experience and accept the sadness of a situation first. Then, I can begin to think more clearly about what my primary responsibility is and how my emotions might affect my thoughts and actions. I will also be sure to inquire openly with patients, families and staff members about how their own personal emotions affect them in similar ways."

\section{Conclusion}

Emotions play a crucial role in moral life. Moral case deliberation can help us to deal with emotions by investigating them and reflecting upon them. Emotions should neither be followed instinctively, nor be discarded and put aside. A proper way of dealing with an emotion is finding the right middle ground between being overwhelmed and remaining untouched. This is not just a matter of rationally determining a balance. One has to be able to act in line with the right middle, and embody the appropriate attitude. Dealing with emotions is a matter of virtue and character. Through the process of 
reflection one becomes aware of the intricacies in learning how to be a better professional and how to respond more openly to the situation.

Addressing emotions in moral case deliberation requires a process of investigation, which is neither driven by empathy, not by moral judgment. Empathy leads to over-involvement with the case presenter. Moral judgment implies that one puts oneself above the case presenter. A true dialogue goes beyond relativism and objectivism (Bernstein 1983). In a dialogue, one learns from the other, not by taking over immediately what the other says, but by investigating the relevance of the experience of the other for one's own situation (Gadamer 1960). In the example, the examination of the emotion of the case presenter led to the conclusion that engagement with the woman should not lead to sharing her sadness, but to showing compassion. Thus, the emotion of the case presenter was put under critique. The criticism was not made from a superior position, but developed through an investigation of the experience of the case presenter and shared by the case presenter. Thus, the group as a whole learned to see the limits of identification and the need for combining personal consideration with professional action.

Reflecting on emotions requires staying close to the emotions. Theoretical elaborations on virtue or excellence do not help to understand what it means to lead a good life and be a good professional. Theoretical discussions go beyond emotions, and make the deliberation process sterile and unfruitful. Reflection on emotions requires being attentive of one's emotions and focusing on the concrete actions to which they give rise. It also requires being open to the fact that others may have different emotions, and be prepared to investigate the differences and learn from them. Thus, learning to deal with emotions is not an individual theoretical exercise, but a joint dialogical investigation into what is important in our daily life.

\section{References}

Abma, T., Molewijk, B., \& Widdershoven, G. (2009). Good care in ongoing dialogue. Improving the quality of care through moral deliberation and responsive evaluation. Health Care Analysis, 17, $217-235$.

Aristotle (1923). Nicomachean ethics, W.D. Ross (Trans.). Oxford: Clarenton Press.

Ashcroft, R., Lucassen, A., Parker, M., Verkerk, M., \& Widdershoven, G. A. M. (Eds.). (2005). Case analysis in clinical ethics. Cambridge: Cambridge University Press.

Bernstein, R. J. (1983). Beyond objectivism and relativism. Oxford: Oxford University Press.

Damasio R. A. (2003). Looking for Spinoza: Joy, sorrow and the feeling brain. New York: Harvest book Harcourt Inc.

Gadamer, H.-G. (1960). Wahrheit und methode. Tübingen: J.C.B. Mohr.

Haidt, J. (2001). The emotional dog and its rational tail: a social intuitionist approach to moral judgment. Psychological Review, 108(4), 814-834.

Kessels, J., Boers, E., \& Mostert, P. (2009). Free space. A field guide for conversations. Amsterdam: Boom Publishers.

Molewijk, B., \& Ahlzen, R. (2011). Clinical ethics committee case 13: Should the school doctor contact the mother of a 17-year-old girl who has expressed suicidal thoughts? Clinical Ethics, 6, 5-10.

Molewijk, B., Abma, T. A., Stolper, M., \& Widdershoven, G. A. M. (2008). Teaching ethics in the clinic. Theory and practice of moral case deliberation. Journal of Medical Ethics, 34, 120-124.

Molewijk, B., Kleinlugtenbelt D., \& Widdershoven, G. (2011). The role of emotions in moral case deliberation: Theory, practice, and methodology. Bioethics, 25(7), 383-393.

Nussbaum, C. M. (2001). Upheavals of thought: The intelligence of emotions. Cambridge: Cambridge University Press. 
Schneider, E. C. (1998). The practice of autonomy: Patients, doctors, and medical decisions. New York: Oxford University Press.

Solbakk, J. H. (2006). Catharsis and moral therapy II: An Aristotelian account. Medicine, Health Care and Philosophy, 9, 141-153.

Steinkamp, N., \& Gordijn, B. (2003). Ethical case deliberation on the ward. A comparison of four models. Medicine, Health care and Philosophy, 6, 235-246.

Walker, M. U. (1998). Moral understandings: A feminist study in ethics. New York: Routledge.

Widdershoven, G., \& Molewijk, B. (2010). Philosophical foundations of clinical ethics: A hermeneutic perspective. Chapter 4. In Gordon Schildmann \& Vollmann (Eds.), Clinical ethics consultation: Theories and methods, implementation, evaluation (pp. 37-52). Williston: Ashgate Publisher. 